\title{
Evidence for Jahn-Teller distortions at the antiferromagnetic transition in $\mathrm{LaTiO}_{3}$
}

\author{
J. Hemberger ${ }^{1}$, H.-A. Krug von Nidda ${ }^{1}$, V. Fritsch ${ }^{1}$, J. Deisenhofer ${ }^{1}$, S. Lobina ${ }^{1}$, \\ T. Rudolf ${ }^{1}$, P. Lunkenheimer ${ }^{1}$, F. Lichtenberg ${ }^{1}$, A. Loidl ${ }^{1}$, D. Bruns ${ }^{2}$, and B. Büchner ${ }^{2}$ \\ ${ }^{1}$ EKM, Institut für Physik, Universität Augsburg, D-86135 Augsburg, Germany \\ ${ }^{2}$ II. Physikalisches Institut A, RWTH Aachen, D-52056 Aachen, Germany
}

\begin{abstract}
$\mathrm{LaTiO}_{3}$ is known as Mott-insulator which orders antiferromagnetically at $T_{\mathrm{N}}=146 \mathrm{~K}$. We report on results of thermal expansion and temperature dependent x-ray diffraction together with measurements of the heat capacity, electrical transport measurements, and optical spectroscopy in untwinned single crystals. At $T_{\mathrm{N}}$ significant structural changes appear, which are volume conserving. Concomitant anomalies are also observed in the dc-resistivity, in bulk modulus, and optical reflectivity spectra. We interpret these experimental observations as evidence of orbital order.
\end{abstract}

PACS numbers: 71.27.+a, 71.30+h, 75.30.Cr, 75.30.Et

In transition-metal oxides a variety of complex electronic ground states is evoked by the interplay of spin, charge, orbital, and lattice degrees of freedom [1]. Amongst those, orbital degeneracy and orbital ordering (OO) have to be considered as key features to understand the physical properties of many compounds. In principle, OO can be detected via neutron diffraction [2, resonant $\mathrm{x}$-ray scattering 3], nuclear magnetic resonance (NMR) [4], or electron spin resonance [5] techniques. However, in most cases lattice distortions induced by $\mathrm{OO}$ phenomena are detected, while the OO parameter and the ground state wave functions remain hidden. In this article we report on structural anomalies in $\mathrm{LaTiO}_{3}$ observed at the antiferromagnetic (AFM) ordering temperature which indicate the onset of $\mathrm{OO}$ induced by spin ordering. The perovskite $\mathrm{LaTiO}_{3}$, where Ti reveals a $3 d^{1}$ electronic configuration, can be characterized as a Mott-Hubbard insulator 6] revealing a $G$-type AFM order below the Néel temperature $T_{\mathrm{N}}=146 \mathrm{~K}[7]$ with an ordered moment of $0.45 \mu_{\mathrm{B}}[8]$.

The orbital ground state in $\mathrm{LaTiO}_{3}$ has not been determined unambiguously: The quasi-cubic crystal field 9 $(\mathrm{CF})$ splits the $3 d$-levels into a low-lying $t_{2 g}$ triplet and an excited $e_{g}$ doublet. The complexity of the problem of a single electron in a threefold degenerate $t_{2 g}$ level, including spin-orbit (SO) coupling and long-range spin order has been outlined long ago by Goodenough [10] and by Kugel and Khomskii 11]. Very recently the orbital ground state of $\mathrm{LaTiO}_{3}$ again came under heavy debate both theoretically $12,13,14,15,16$ and experimentally [4, 17, 18, 19, 20]. Khaliullin and Maekawa 12 proposed the scenario of an orbital liquid to explain the reduced magnetic moment and the small spin-wave anisotropy observed in neutron-scattering experiments 18. They predicted a gap of the orbital excitations, which however could not be observed experimentally [17]. To solve this discrepancy a refined model has been proposed, which may explain the dominant magnon contribution to the specific heat [16]. Mochizuki and Imada [15] investigated several scenarios that lift the $t_{2 g}$ degeneracy based on the competition of SO coupling, Jahn-Teller (JT) effect, and
$\mathrm{CF}$ due to $\mathrm{GdFeO}_{3}$-type lattice distortions for $R \mathrm{TiO}_{3}$ with $(R=\mathrm{La}, \mathrm{Sm}, \mathrm{Nd}, \mathrm{Gd}, \mathrm{Y})$. While in $\mathrm{YTiO}_{3}$ the JT distortion was shown to be dominant, the influence of SO coupling must be considered important for $\mathrm{LaTiO}_{3}$ $[14,15]$. Also the experimental situation is not yet settled. Itoh et al. 44 have explained their NMR results in $\mathrm{LaTiO}_{3}$ assuming a degenerate orbital ground state. Neutron scattering provided no experimental evidence for OO but has been interpreted in terms of an orbital liquid 18]. However, an anisotropy of the magnetization was observed as well in the paramagnetic (PM) as in the AFM regime in Ref. 17. A small JT distortion has been derived from the observation of atomic displacements by means of transmission electron microscopy [19] and, utilizing X-ray and neutron diffraction, the observation of structural anomalies at $T_{\mathrm{N}}$ was reported recently [20].

The purpose of this work is to give a detailed structural characterization of $\mathrm{LaTiO}_{3}$, including thermal expansion and phonon properties and to relate these to electronic, thermodynamic, and magnetic properties. The observed anomalies are interpreted in terms of $\mathrm{OO}$.

Untwinned single crystals of $\mathrm{LaTiO}_{3}$ have been prepared by floating-zone melting as described elsewhere 7]. The oxygen content was determined by thermogravimetry. The x-ray diffraction pattern at $T=295 \mathrm{~K}$ revealed an orthorhombic structure $(\mathrm{P} b n m, z=4)$ with the lattice parameters $a=5.633 \AA, b=5.617 \AA, c=7.915 \AA$. Laue measurements were performed on the single crystalline samples to orient the samples and to exclude twinning. The electrical resistivity was measured with a four-probe electrometer circuit in the temperature range $30 \leq T \leq 300 \mathrm{~K}$. The specific heat was obtained with noncommercial setups utilizing a quasi-adiabatic method between $2 \mathrm{~K}$ and $15 \mathrm{~K}$ and an AC-method between $10 \mathrm{~K}$ and $300 \mathrm{~K}$. X-ray powder diffraction was performed in the temperature range $90 \leq T \leq 350 \mathrm{~K}$ employing a STOE diffractometer with a nitrogen gas-flow cryostat. The linear thermal expansion coefficient $\alpha=(\partial L / \partial T) / L$ was determined utilizing a home-built high-resolution capacitance dilatometer. The measurements of the optical reflectivity were carried out on polished single crystals us- 


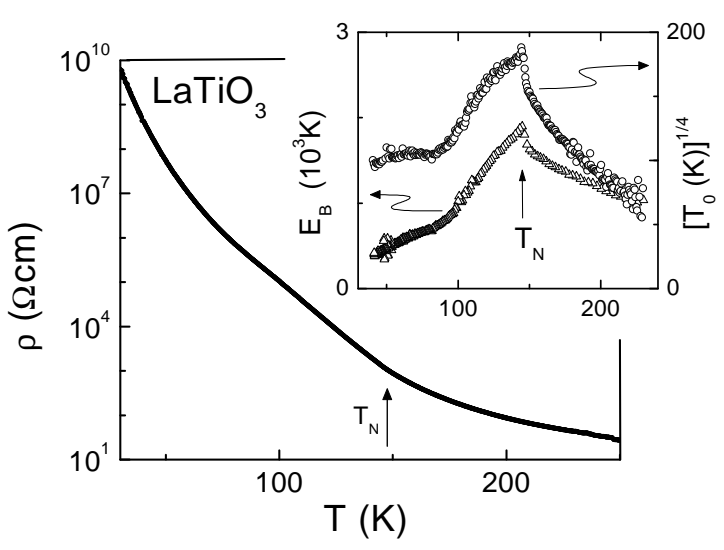

FIG. 1: DC-resistivity of $\mathrm{LaTiO}_{3}$. The inset shows the $T$ dependence of the effective energy barrier assuming an Arrhenius-type behavior $\rho=\rho_{0} \exp \left(E_{\mathrm{B}} / T\right)$ (left scale) or the characteristic temperature of 3-dimensional VRH $\rho \propto$ $\exp \left[\left(T_{0} / T\right)^{1 / 4}\right]$ (right scale) as described in the text.

ing a Fourier transform IR spectrometer (BRUKER IFS $113 \mathrm{v})$.

Figure 1 displays measurements of the DC-resistivity. At all temperatures, above and below $T_{\mathrm{N}}$, the resistivity $\rho(T)$ exhibits semiconducting characteristics. The arrow marks the magnetic transition at $T_{\mathrm{N}}=146 \mathrm{~K}$ determined from susceptibility measurements 17 for the sample under investigation. It is worth mentioning that this temperature corresponds to the highest transition temperatures reported in literature [7, 9]. It is known that $\mathrm{LaTiO}_{3}$ is sensitive to deviations from its nominal stoichiometry 7, 21, 22, 23, 24], driving the Mott-Hubbardinsulator away from half filling towards a metallic state yielding a concomitant strong reduction of $T_{\mathrm{N}}$. Thus, the high resistivity and the high transition temperature show that the samples under investigation are close to the nominal composition. The inset of Fig. 1 gives a representation according to the assumption of thermally activated transport and variable range hopping (VRH), respectively. The derivatives $E_{B}=\mathrm{d}(\ln \rho) / \mathrm{d}(1 / T)$ (left scale) and $T_{0}^{1 / 4}=\mathrm{d}(\ln \rho) / \mathrm{d}\left(1 / T^{1 / 4}\right)$ (right scale) are plotted versus temperature. These quantities can be interpreted as generalized energy barrier $E_{B}$ in the case of purely activated transport or as characteristic temperature $T_{0}$ assuming VRH. Both curves reveal a strong variation from far above to well below the magnetic ordering transition with a spike-like enhancement just at $T_{\mathrm{N}}$. From the inset in Fig. 1 it is clear that neither a purely thermally activated behavior nor 3-dimensional (3D) hopping transport can account for the $T$ dependence of the resistivity in $\mathrm{LaTiO}_{3}$. At this point we propose that not only spin-dependent scattering processes are responsible for the anomaly of the resistivity around $T_{\mathrm{N}}$, but rather that orbital fluctuations have to be taken into account. It is reasonable to assume that the inter-

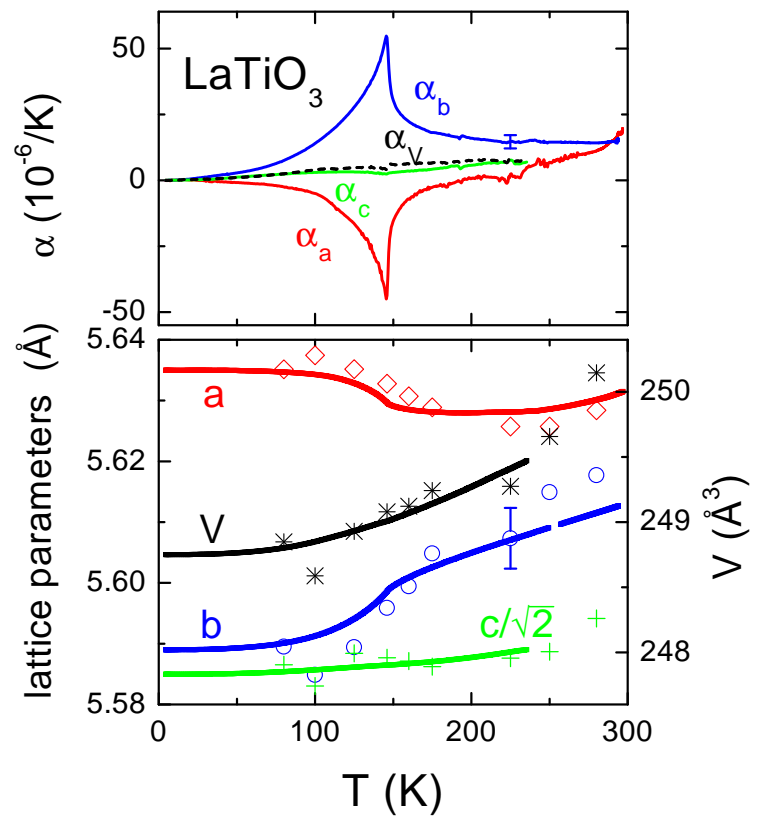

FIG. 2: Upper frame: Thermal expansion coefficients of $\mathrm{LaTiO}_{3}$ for the three different crystallographic directions. The dashed line gives the averaged volume expansion coefficient $\alpha_{V}=\left(\alpha_{a}+\alpha_{b}+\alpha_{c}\right) / 3$. Lower frame: $T$ dependence of the lattice parameters $a, b, c / \sqrt{2}$ (left scale), and cell volume $V$ (right scale). The symbols denote data obtained by x-ray diffraction. The solid lines were calculated by the integration of $\alpha$ and scaled to the lattice constants. The error bar given in the lower frame refers only to the uncertainty of the absolute values gained by the scaling procedure. The relative resolution is by far higher.

site transfer matrix elements, responsible for the charge transport, could strongly be influenced by the orbital dynamics. It is also interesting to note that below $100 \mathrm{~K}$, 3D-VRH dominates the charge transport, demonstrated by the temperature independent value of the respective derivative shown in the inset of Fig. [1 For temperatures $T \leq 100 \mathrm{~K}$ the derivative approaches a constant value with $T_{0} \approx 10^{8} \mathrm{~K}$, which is close to those values observed in doped manganites 25], where OO is established.

Looking for further indications of $\mathrm{OO}$ close to $T_{\mathrm{N}}$, we investigated the $T$ dependence of the linear thermal expansion coefficient $\alpha=(\partial L / \partial T) / L$ for $\mathrm{LaTiO}_{3}$. The results for the different crystallographic directions are shown in the upper frame of Fig. 2 At the AFM transition a sharp peak-like anomaly can be detected along $a$ and $b$ direction. In contrast, the magnetic transition can hardly be detected in the measurements along $c$. The thermal expansion coefficient along the $a$ - and $b$-direction reaches values up to $|\alpha|=5 \times 10^{-5} \mathrm{~K}^{-1}$, about ten times higher than e.g. those near the displacive phase transition in non-magnetic $\mathrm{SrTiO}_{3}$ at $107 \mathrm{~K}$ [26]. But both directions $a$ and $b$ have opposite sign: while $\alpha_{b}$ is positive for all temperatures, $\alpha_{a}$ is negative for $T<200 \mathrm{~K}$. Obviously, the crystal expands along the 
$a$-direction with decreasing temperature. At the same time, the averaged volume thermal expansion coefficient $\alpha_{V}=\left(\alpha_{a}+\alpha_{b}+\alpha_{c}\right) / 3$ (dashed line in Fig. 22) exhibits only a weak increase with temperature as expected for ordinary solids and no anomaly can be found within the uncertainty of the measurement, i.e. the structural anomaly at $T_{\mathrm{N}}$ is volume conserving. It is remarkable, that the deviations of $\alpha_{a}$ and $\alpha_{b}$ from $\alpha_{V}$ set in already well above $T_{\mathrm{N}}$. While the measurements of the thermal expansion allow a much higher relative resolution compared to diffraction methods, it is difficult to derive absolute values of the lattice parameters from the dilatation data. For this reason we combined these results with temperature dependent x-ray diffraction measurements down to $T=70 \mathrm{~K}$. The results for the $T$ dependence of the lattice constants (left scale) and the volume of the unit cell (right scale) are displayed in the lower frame of Fig. 2. Again it is remarkable that the cell volume $V(T)$ reveals no anomaly at $T_{\mathrm{N}}$. The same is valid for $c(T)$. The lattice parameters $a(T)$ and $b(T)$ exhibit distinct anomalies with opposite signs. However, even though the difference between $a$ and $b$ is strongly increased and $b$ nearly equals $c / \sqrt{2}$ for low temperatures, the orthorhombic crystal symmetry is not changed. We interpret these phenomena as an isostructural orbital order-disorder transition resulting from $\mathrm{OO}$ similar to the case of $\mathrm{LaMnO}_{3}[27,28]$.

In perovskites the orthorhombic $O^{\prime}$ phase with $a>$ $c / \sqrt{2}$ points towards a cooperative JT distortion. Although the lattice parameters of $\mathrm{LaTiO}_{3}$ at room temperature already fulfill this condition, to the best of our knowledge the JT effect has never been considered in the PM regime in this compound, but is very likely to appear in the magnetically ordered phase: Following Goodenough [10], the observed distortions around $T_{N}$ can be understood in terms of the energy-level scheme constructed from the $t_{2 g}$ states which resemble p-like electron states (effective orbital moment $\tilde{L}=1$ ). SO coupling and the low symmetry component of the $\mathrm{CF}$ split this orbital triplet, which is occupied by one electron (spin $S=1 / 2$ ), into three Kramers doublets even above $T_{N}$. In the perovskite structure magnetic order is usually found to initiate the JT effect in the $t_{2 g}$ levels [10], whereas this is not necessarily the case for the $e_{g}$ levels like for example in $\mathrm{LaMnO}_{3}\left(T_{\mathrm{N}}=140 \mathrm{~K}\right)$, where the cooperative JT effect persists up to $750 \mathrm{~K}$ and at $T_{N}$ only an additional enhancement of spin-phonon coupling can be observed 28]. Hence, in $\mathrm{LaTiO}_{3}$, we interpret the observed structural anomalies at $T_{\mathrm{N}}$ in terms of a cooperative JT distortion as expected for the $t_{2 g}$ levels just below the magnetic transition in magnets with collinear spin order. The underlying OO can be understood taking into account the $p$-wave symmetry of the $\tilde{L}=1$ manifold. A $p$-like orbital induces a uniaxial elongation of a $\mathrm{TiO}_{6}$ octahedron along two opposite $\mathrm{Ti}-\mathrm{O}$ bonds. In the ground-state the $p$-like orbitals are fixed along this $\mathrm{O}-\mathrm{Ti}-\mathrm{O}$ direction within the $a b$ plane and order in a fer-

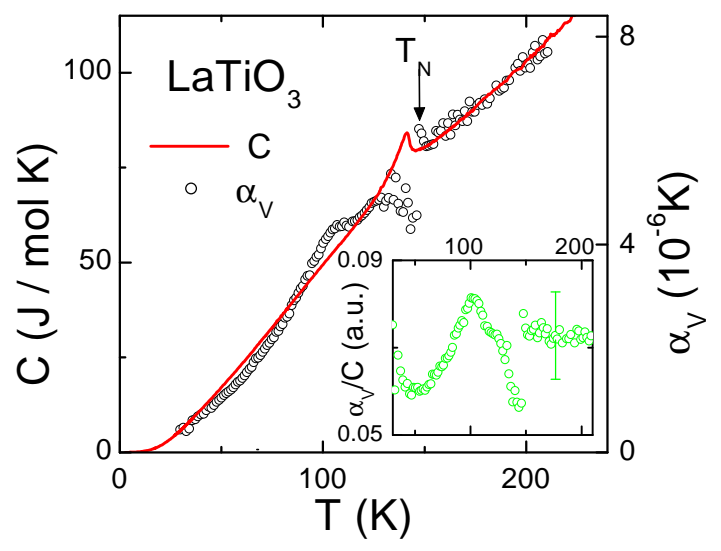

FIG. 3: Specific heat (left scale) and volume thermal expansion coefficient (right scale) of $\mathrm{LaTiO}_{3}$. Lower inset: Volume Grüneisenparameter $\Gamma_{V} \propto \alpha_{V} / C$ averaged from the three crystallographic directions devided by the bulk modulus $B$.

rodistorsive pattern induced by the $G$-type AFM spin order. This amplifies the weak orthorhombic distortion, which (possibly together with orbital fluctuations) is already present in the PM phase, resulting in the experimentally observed expansion along the crystallographic $a$ direction.

The $T$ dependence of the specific heat $C$ of $\mathrm{LaTiO}_{3}$ is displayed in Fig. 3 together with the averaged thermal expansion coefficient. The behavior of $C(T)$ coincides very well with that of $\alpha_{V}$ above the magnetic transition, but exhibits deviations at and below $T_{\mathrm{N}}$. These deviations can better be illustrated in terms of the thermodynamic Grüneisenparameter $\Gamma=3 \alpha_{V} V B / C$. In the inset of Fig. 3 we show the $T$ dependence of the ratio of the volume thermal expansion coefficient and the heat capacity, $\alpha_{V} V / C \propto \Gamma / B$. In normal anharmonic crystals $\Gamma$ is only weakly temperature dependent and the bulk modulus should slightly increase with decreasing temperature. However, it is also well known that the acoustic phonons directly couple to the orbital (quadrupolar) degrees of freedom and, hence, the bulk modulus should be sensitive to orbital fluctuations and to the onset of OO. One example of the softening of longitudinal acoustic modes at the JT transition in doped manganites can be found in Ref. 25. For $T>T_{\mathrm{N}}$, the ratio $\Gamma / B \propto \alpha_{V} V / C$ is nearly constant. It reveals a distinct and strong anomaly at $T_{\mathrm{N}}$, is strongly enhanced just below the ordering temperature and subsequently passes through a minimum on further decreasing temperature. The minimum at low temperatures can be explained by magnetic excitations [17], which will strongly affect the specific heat, but much less the thermal expansion. It seems straightforward to explain the maximum in $\Gamma / B$ just below $T_{\mathrm{N}}$ by the onset of OO. As has been documented in the manganites, the longitudinal acoustic modes, and hence also the bulk modulus, reveal a significant softening at the JT transi- 


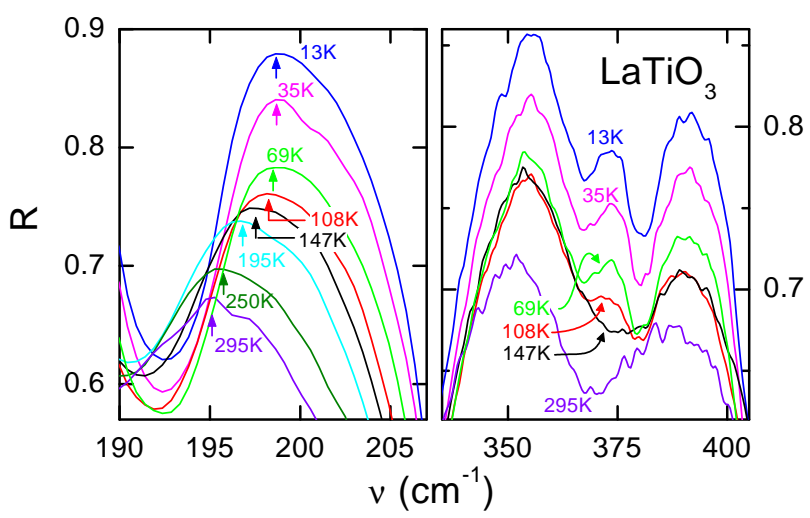

FIG. 4: Spectra of the optical reflectivity for various temperatures. The data illustrates the $T$ dependence of selected phonon modes.

tion 25].

Finally we will show that in the AFM phase also the phonon spectrum is strongly influenced, indicating significant changes of the local structure and the binding energies. At room temperature the reflectance of $\mathrm{LaTiO}_{3}$ shows the three characteristic bands of phonons of the perovskite structure grouped around 200, 400, and 600 $\mathrm{cm}^{-1}$, which roughly can be ascribed to external, bending, and stretching modes 23]. In cubic symmetry only three modes are IR active. In the orthorhombic Pnma structure these bands split into single phonon excitations and 25 infrared-active modes have to be expected [29]. Fig. 4 shows the reflectance in $\mathrm{LaTiO}_{3}$ for two selected groups of modes close to 200 and $400 \mathrm{~cm}^{-1}$ for various temperatures. A detailed report on the optical properties will be given in a forthcoming paper [30]. The phonons at $200 \mathrm{~cm}^{-1}$ belong to the external modes were the $\mathrm{TiO}_{6}$ octahedra vibrate against the La ions. The modes close to $400 \mathrm{~cm}^{-1}$ can be characterized as Ti-O bond-angle modulations. Without going into further details, already the raw data provide striking experimental evidence that close to $T_{\mathrm{N}}$ the external modes reveal a significant shift of the eigenfrequencies (indicated by arrows). At $T_{\mathrm{N}}$ these modes considerably stiffen on decreasing temperatures. In the group of bending modes, below $T_{\mathrm{N}}$ one mode significantly increases in the spectra at $375 \mathrm{~cm}^{-1}$ indicating that the local symmetry at $T_{\mathrm{N}}$ changes considerably.

In conclusion, we have presented detailed measurements of the electrical resistivity, the $T$ dependence of lattice constants, thermal expansion, and heat capacity, as well as of the optical reflectivity for selected groups of phonons in stoichiometric single crystalline and untwinned $\mathrm{LaTiO}_{3}$. All results exhibit anomalies slightly above or just at the magnetic ordering temperature indicating significant structural changes. At this transition the PM and orthorhombic crystal characterized by lattice constants $a>b>c / \sqrt{2}$ transforms into an antiferromag- net with almost tetragonal symmetry $(a>b \approx c / \sqrt{2})$. We interpret these significant structural changes as evidence of OO. Both the PM and the AFM phase can be consistently described assuming a splitting of the $t_{2 g}$ state into three Kramers doublets. Below $T_{N}$ the $G$-type AFM order induces an additional cooperative JT distortion, which results in a ferrodistortive OO pattern. Even so it can not be ruled out, if additional quantum fluctuations 14] have to be taken into account to describe the orbital groundstate, it is difficult to explain such type of structural distortion related to the onset of magnetic order within a picture of entirely obliterated orbital order as proposed in terms of the orbital-liquid scenarios.

This work was supported by the BMBF via VDI/EKM, FKZ 13N6917/18 and by the DFG within SFB 484 (Augsburg). We are grateful to M. V. Eremin for useful discussions.

[1] Y. Tokura and N. Nagaosa, Science 288, 462 (2000).

[2] J. Rodriguez-Carvajal et al., Phys. Rev. B 57, R3189 (1998).

[3] Y. Murakami et al., Phys. Rev. Lett. 80, 1932 (1998).

[4] M. Itoh et al., J. Phys. Soc. Jpn. 68, 2783 (1999).

[5] J. Deisenhofer et al., Phys. Rev. B 65, 104440 (2002).

[6] Y. Okimoto et al., Phys. Rev. B 51, 9581 (1995).

[7] F. Lichtenberg et al., Z. Phys. B 82, 211 (1991).

[8] J.P. Goral and J.E. Greedan, J. Magn. Magn. Mater. 37, 315 (1983).

[9] G. I. Meijer et al., Phys. Rev. B 59, 11832 (1999).

[10] J.B. Goodenough, Phys. Rev. 171, 466 (1968).

[11] K.I. Kugel and D. Khmoskii, Sov. Phys. Usp. 25, 231 (1982)

[12] G. Khaliullin and S. Maekawa, Phys. Rev. Lett. 85, 3950 (2000).

[13] G. Khaliullin, Phys. Rev. B 64, 212405 (2001).

[14] G. Khaliullin and S. Okamoto, Phys. Rev. Lett. 89, 167201 (2002).

[15] M. Mochizuki and M. Imada, J. Phys. Soc. Jpn. 70, 2872 (2001); ibid. 1777 (2001); cond-mat/0301049 v1.

[16] K. Kikoin et al., cond-mat/0302182

[17] V. Fritsch et al., Phys. Rev. B 65, 212405 (2002).

[18] B. Keimer et al., Phys. Rev. Lett. 85, 3946 (2000).

[19] M. Arao, Y. Inoue, and Y. Koyama, J. Phys. Chem. Sol. 63, 995 (2002).

[20] M. Cwik et al., cond-mat/0302087

[21] Y. Okada et al., Phys. Rev. B 48, 9677 (1993).

[22] Y. Taguchi et al., Phys. Rev. B 59, 7917 (1999).

[23] D.A. Crandles et al., Phys. Rev. B 49, 16207 (1994).

[24] V. Fritsch et al., Phys. Rev. B 64, 045113 (2001).

[25] M. Paraskevopoulos et al., J. Phys.: Condens. Matter 12, 3993 (2000).

[26] H. Willemsen et al., Phys. Rev. B 14, 3644 (1976).

[27] M.C. Sánchez et al., Phys. Rev. Lett. 90, 045503 (2003).

[28] E. Granado et al., Phys. Rev. B 60, 11879 (1999).

[29] F. Mayr et al., Phys. Rev. B 62, 15673 (2000).

[30] P. Lunkenheimer et al., cond-mat/0307333 (submitted to Phys. Rev. B) 